Original paper

\title{
Evaluation of a cycle-generative adversarial network-based cone-beam CT to synthetic CT conversion algorithm for adaptive radiation therapy
}

\author{
Miriam Eckl $^{\text {a, } 1}$, Lea Hoppen ${ }^{\text {a, }, 1}$, Gustavo R. Sarria ${ }^{\text {b }}$, Judit Boda-Heggemann ${ }^{\text {a, }}$ \\ Anna Simeonova-Chergou ${ }^{a}$, Volker Steil ${ }^{a}$, Frank A. Giordano ${ }^{b}$, Jens Fleckenstein ${ }^{a}$ \\ ${ }^{a}$ Department of Radiation Oncology, University Medical Center Mannheim, University of Heidelberg, Germany \\ ${ }^{\mathrm{b}}$ Department of Radiology and Radiation Oncology, University Hospital Bonn, Germany
}

\section{A R T I C L E I N F O}

\section{Keywords:}

Synthetic CT

Cone-beam CT

Cycle-generative adversarial network-based

image correction

Adaptive radiation therapy

\begin{abstract}
A B S T R A C T
Purpose: Image-guided radiation therapy could benefit from implementing adaptive radiation therapy (ART) techniques. A cycle-generative adversarial network (cycle-GAN)-based cone-beam computed tomography (CBCT)-to-synthetic CT (sCT) conversion algorithm was evaluated regarding image quality, image segmentation and dosimetric accuracy for head and neck $(\mathrm{H} \& \mathrm{~N})$, thoracic and pelvic body regions.

Methods: Using a cycle-GAN, three body site-specific models were priorly trained with independent paired CT and CBCT datasets of a kV imaging system (XVI, Elekta). sCT were generated based on first-fraction CBCT for 15 patients of each body region. Mean errors (ME) and mean absolute errors (MAE) were analyzed for the sCT. On the sCT, manually delineated structures were compared to deformed structures from the planning CT (pCT) and evaluated with standard segmentation metrics. Treatment plans were recalculated on sCT. A comparison of clinically relevant dose-volume parameters $\left(D_{98}, D_{50}\right.$ and $D_{2}$ of the target volume) and 3D-gamma $(3 \% / 3 \mathrm{~mm})$ analysis were performed.

Results: The mean ME and MAE were 1.4, 29.6, 5.4 Hounsfield units (HU) and 77.2, 94.2, $41.8 \mathrm{HU}$ for $\mathrm{H} \& \mathrm{~N}$, thoracic and pelvic region, respectively. Dice similarity coefficients varied between $66.7 \pm 8.3 \%$ (seminal vesicles) and $94.9 \pm 2.0 \%$ (lungs). Maximum mean surface distances were $6.3 \mathrm{~mm}$ (heart), followed by $3.5 \mathrm{~mm}$ (brainstem). The mean dosimetric differences of the target volumes did not exceed 1.7\%. Mean 3D gamma pass rates greater than $97.8 \%$ were achieved in all cases.

Conclusions: The presented method generates SCT images with a quality close to pCT and yielded clinically acceptable dosimetric deviations. Thus, an important prerequisite towards clinical implementation of CBCTbased ART is fulfilled.
\end{abstract}

\section{Introduction}

Adaptive radiation therapy (ART) is one of the latest improvements in radiation therapy treatment quality. Currently, the conventional linac-based radiation therapy workflow neglects morphological changes during fractionated treatment courses. By applying ART techniques, daily imaging could not only be used for translational and rotational patient positioning but also for modifying the initial treatment plan to compensate for a deformed patient anatomy [1-4]. Interfractional treatment adaptations following CBCT based image guidance are common in the head and neck (H\&N), thoracic and pelvic region [5] due to random organ motion or deformations (weight loss, tumor shrinkage, etc.) [6].

For conventional linear accelerator (linac)-based radiation therapy a major challenge on the way towards CBCT-based ART is insufficient cone-beam computed tomography (CBCT) image quality [7-9]. A CBCT possesses image artifacts due to detector scatter, patient specific scatter, image lag and beam hardening, making dose calculations prone to errors. Many calibration approaches for accurate dose calculations on CBCT currently exist [10]: Patient- or population-specific CT number to electron density calibration (CT-ED-calibration) [11], bulk density override [12], image processing algorithms that further improve the

\footnotetext{
* Corresponding author at: Department of Radiation Oncology, University Medical Center Mannheim, University of Heidelberg, Theodor-Kutzer Ufer 1-3, 68167 Mannheim, Germany.

E-mail address: Lea.Hoppen@umm.de (L. Hoppen).

1 The first and second author contributed equally to this work.
} 
image quality [13-15] or deformable image registration (DIR) $[7,8]$ between the CBCT and the treatment planning CT (pCT) to create a deformed CT or a deformed dose in order to assess anatomical and dosimetric changes [16,17]. Recently, deep learning (DL) approaches gained popularity since they can be custom tailored to individual treatment components like image processing and can substantially accelerate time-consuming workflow steps in ART [18,19]. Artificial neural networks can be trained with multimodal datasets (e.g. CT and CBCT or CT and magnetic resonance images (MRI)) and preserve the anatomy of the daily images in the derived synthetic CT images (sCT). Several studies achieved a significant artifacts reduction and therefore increased the potential for a clinical implementation of ART for different anatomical regions [20-25].

The aim of this work is to analyze a cycle-generative adversarial network (cycle-GAN)-based CBCT-to-sCT conversion algorithm for three different anatomical regions ( $\mathrm{H} \& \mathrm{~N}$, thorax and pelvis). Each essential step of an ART workflow was evaluated for the generated SCT of the first treatment fraction $\mathrm{CBCT}$ in comparison to the pCT with respect to answering the following questions:

(1) Are the image uncertainties of an sCT similar to those of a pCT?

(2) Is the contour propagation on the SCT of comparable quality to the manually created contours or can it at least be modified while the patient remains on the couch during treatment?

(3) Is the dosimetric accuracy of similar quality compared to the pCT?

\section{Material and methods}

\subsection{Patient population and treatment workflow}

A total of 45 radiation therapy patients, comprising $15 \mathrm{H} \& \mathrm{~N}, 15$ thoracic and 15 pelvic target volumes were included and retrospectively analyzed after IRB approval (2018-836R-MA). These testing datasets were entirely different from the cycle-GAN model training datasets. Both training and testing datasets share the same patient demographics which are presented in Table 1. Patients in prone position or with metallic implants other than dental implants were excluded from the study.

pCT images were acquired with a CT scanner (Brilliance Big Bore, Philips Healthcare, Amsterdam, Netherlands) with the body site-specific scan protocols displayed in Table 2. Scanner-specific CT-ED-calibration curves were applied to both the pCT and the SCT datasets. The standard deviation (SD) of the pCT's image noise was obtained with a tissuecharacterization phantom (model 467, Gammex, Middleton, WI, USA) and scan protocols from Table 2. Monte Carlo based dose calculations in Monaco 5.11 (Elekta AB, Stockholm, Sweden) were performed with a grid size of $2 \mathrm{~mm}$ and a statistical uncertainty of $0.5 \%$ on both pCT and sCT.

Patients were treated on a linac (VersaHD, Elekta $\mathrm{AB}$ ) and daily kVbased CBCT (XVI, Elekta AB) were obtained prior to each treatment fraction, reconstructed and exported after rigid registration to the $\mathrm{PCT}$ with a slice thickness of $3 \mathrm{~mm}$. Detailed СBCT acquisition parameters are shown in Table 2.
For the sCT generation step, no DIR between the CBCT and the pCT was performed. To minimize anatomical differences to the $\mathrm{pCT}$, only the first treatment fraction CBCT was used for SCT generation for each patient in the following analysis. The average time between the pCT and the first fraction СВСТ was $5 \pm 2.1$ days, $3.6 \pm 1.5$ days and $4 \pm 2.3$ days for the $H \& N$, thorax and pelvis models.

\subsection{The cycle-GAN architecture}

The research software ADMIRE (ADvanced Medical Image Registration Engine) (Elekta $\mathrm{AB}$ ) uses a 2D cycle-GAN to provide DIR-based automated image segmentation and to generate sCT based on CBCT (XVI, Elekta $\mathrm{AB}$ ) in a standalone module of the treatment planning system Monaco. The two network structures, generators and discriminators used in the presented study were similar to the ones in the original network $[26,27]$. Two main issues of applying the original cycleGAN on medical image synthesis are that the synthesized images may have some level of geometry or structure distortion and may not achieve pixel-level accuracy because of the mere distribution match in two domains [28]. In order to address these issues, additional modifications of the original cycle-GAN network were implemented by Elekta and are part of ADMIRE. Firstly, different to the unpaired training mechanism adopted in several previous research works $[22,29,30]$, the training strategy in this sCT module is based on paired CBCT and pCT. The paired pCT were obtained by applying a DIR in ADMIRE on the original pCT. Secondly, besides the original adversarial loss and cycle loss terms, an L1 penalty term between the sCT and the corresponding paired images for selected areas was employed for both "forward" and "backward" direction. Thirdly, transform layers were added into the original networks to suppress the potential structure distortion. The training module and deployment module were implemented by Tensorflow library with Python and $\mathrm{C}++$ interfaces.

Three specific models were trained separately by Elekta for three body regions: pelvis, thorax and H\&N. For the pelvis and thorax model, a total of 205 and 53 paired CBCT-and-CT patient volumes from different patients were collected, respectively. For the H\&N model, a total of 120 paired volumes were collected from 25 different patients with four or five daily CBCT each. The training datasets for the pelvic body region were collected at seven different clinical centers and at one center for the thoracic and $\mathrm{H} \& \mathrm{~N}$ body region. All images were acquired with identical scan protocols that are listed in Table 2.

In order to have better representation for high density structures like bones or teeth, all paired CBCT and CT used in thorax and H\&N regions were clipped into the value range [-1000 4000] Hounsfield units (HU), while those for pelvis model were clipped into the value range $[-1000$ $1000]$ HU. All images were linearly scaled into [-1.0 1.0] as the input and predicted output for all training procedures. For standardization and to reduce training time, all images were resampled to a size of 224 $\times 224$ pixels. In terms of training procedure, an Adam solver with the initial learning rate of 0.0002 was adopted and a total of 200 epochs were performed. The learning rate was fixed for the first 100 epochs and afterwards linearly decayed. All hyperparameters were originally implemented by Elekta in a standalone module and thus remained

Table 1

Patient characteristics of the training and evaluation datasets.

\begin{tabular}{|c|c|c|c|c|c|c|c|}
\hline $\begin{array}{l}\text { Body } \\
\text { site }\end{array}$ & $\begin{array}{l}\text { Patients for } \\
\text { training / } \\
\text { evaluation }\end{array}$ & Diagnosis & $\begin{array}{l}\text { Tumor } \\
\text { stage }\end{array}$ & $\begin{array}{l}\text { Energy (MV) and } \\
\text { treatment modality } \\
\text { (VMAT or DMLC) }\end{array}$ & $\begin{array}{l}\text { Prescription } \\
\text { dose (Gy) }\end{array}$ & $\begin{array}{l}\text { Fraction } \\
\text { dose (Gy) }\end{array}$ & $\begin{array}{l}\text { Average patient age and } \\
\text { range for training/ } \\
\text { evaluation (years) }\end{array}$ \\
\hline $\mathrm{H} \& \mathrm{~N}$ & $25 / 15$ & $\begin{array}{l}\text { Squamous cell cancer, oropharynx } \\
\text { cancer, larynx cancer, tonsil cancer, } \\
\text { lymph node cancer }\end{array}$ & $\mathrm{T} 2-\mathrm{T} 4 \mathrm{~b}$ & 6, VMAT or DMLC & $50,60,70$ & 2 & $61.7(54-80) / 68.9(58-86)$ \\
\hline Thorax & $53 / 15$ & $\begin{array}{l}\text { Upper and middle lobe lung cancer, } \\
\text { intrathoracic lymph node cancer }\end{array}$ & T2b-T4a & $\begin{array}{l}\text { 10, VMAT or DMLC } \\
\text { (with flattening filter) }\end{array}$ & $60,66,70$ & 2 & $66.1(51-90) / 62.5(55-84)$ \\
\hline Pelvis & $205 / 15$ & $\begin{array}{l}\text { Malignant neoplasm of prostate } \\
\text { (including seminal vesicles) }\end{array}$ & T1c-T2c & 10, VMAT & 60 & 2 or 3 & $71.6(50-82) / 73.6(57-84)$ \\
\hline
\end{tabular}


Table 2

Image acquisition parameters of CT and cone-beam CT (СВСТ).

\begin{tabular}{|c|c|c|c|c|c|c|c|c|c|}
\hline Modality & $\begin{array}{l}\text { Body } \\
\text { site }\end{array}$ & $\begin{array}{l}\text { Field-of-view }(\mathrm{cm} \times \\
\mathrm{cm})\end{array}$ & Matrix & $\begin{array}{l}\text { Slice thickness } \\
(\mathrm{mm})\end{array}$ & $\begin{array}{l}\text { Voltage } \\
(\mathrm{kV})\end{array}$ & $\begin{array}{l}\text { Current * time } \\
(\mathrm{mAs})\end{array}$ & $\begin{array}{l}\text { Acquisition time } \\
\text { (s) }\end{array}$ & $\begin{array}{l}\text { Filter, } \\
\text { collimator }\end{array}$ & $\begin{array}{l}\text { Acquisition interval } \\
\left({ }^{\circ}\right)\end{array}$ \\
\hline \multirow[t]{3}{*}{ СT } & $\mathrm{H} \& \mathrm{~N}$ & & 512 & 3 & 120 & 116 & 32.8 & Standard (B) & \\
\hline & Thorax & & 512 & 3 & 120 & 116 & 13 & Standard (B) & \\
\hline & Pelvis & & 512 & 3 & 120 & 145 & 13 & Standard (B) & \\
\hline \multirow[t]{3}{*}{ СВCT } & $\mathrm{H} \& \mathrm{~N}$ & $27.6 \times 27.6$ & 512 & 3 & 100 & 18.3 & 68 & F0 (empty), S20 & 40 to -160 \\
\hline & Thorax & $27.6 \times 42.6$ & 512 & 3 & 120 & 132 & 120 & $\begin{array}{l}\text { F1 (bow-tie), } \\
\text { M20 }\end{array}$ & -180 to 180 \\
\hline & Pelvis & $27.6 \times 42.6$ & 512 & 3 & 120 & 132 & 120 & $\begin{array}{l}\text { F1 (bow-tie), } \\
\text { M20 }\end{array}$ & -180 to 180 \\
\hline
\end{tabular}

unaltered for the evaluation of the three body region models.

\subsection{Analysis of image uncertainties}

A total of seven regions of interest (ROI) were generated on the sCT to evaluate the site-specific model accuracy within specific CT number regions. An expert physician manually delineated the patient outline for the "body contour ROI", lungs for the "lung ROI", bladder, prostate and rectum for the "pelvic soft tissue ROI", pelvic and femoral bones for the "pelvic bone ROI" and mandible and cranial bones for the "H\&N bone ROI" on the sCT. In order to compensate for possible anatomical differences between pCT and SCT negative margins were applied (solely for image quality analysis) to the body contour ROI (15 mm), lung ROI and pelvic soft tissue ROI (10 mm), the pelvic bone ROI ( $4 \mathrm{~mm}$ ) and the H\&N bone ROI $(2 \mathrm{~mm})$. The body contour ROI was evaluated for all three cycle-GAN models. For the thorax model the lung ROI, for the pelvis model the pelvic soft tissue ROI and pelvic bone ROI and for H\&N model the H\&N bone ROI were additionally examined. In order to assess the image quality the mean error (ME) and mean absolute error (MAE) between sCT and pCT for all ROIs were calculated as

$\operatorname{ME}(X, Y)=\frac{1}{N} \sum_{i=1}^{N} X_{i}-Y_{i}$,

$\operatorname{MAE}(X, Y)=\frac{1}{N} \sum_{i=1}^{N}\left|X_{i}-Y_{i}\right|$

with $\mathrm{N}$ denoting the number of voxels within a ROI, $\mathrm{X}_{\mathrm{i}}$ is the $\mathrm{i}$-th greyvalue of sCT and $Y_{i}$ is the $i$-th pixel-value of the CT in HU. Furthermore, a two-tailed $t$ test between the mean of the obtained ME values and $\mathrm{OHU}$ was performed.

\subsection{Image segmentation accuracy analysis on $s C T$}

To evaluate whether differences in image quality in pCT and sCT affect the image segmentation accuracy, manually created structures from the pCT were transferred via a DIR to the sCT and compared to structures that were directly generated on SCT. Therefore, an expert physician manually delineated the clinical target volume (CTV) and the following organs at risk (OAR) on pCT and sCT: Brainstem, spinal cord, parotid glands, plexus, lungs, heart, bladder, prostate, seminal vesicles and rectum. Subsequently, the intra-patient segmentation module in ADMIRE was used to perform a DIR between the pCT and the SCT. This DIR consists of three registration steps that gradually increase the freedom for image matching: A global mutual information based rigid registration, a block-wise normalized-sum-of-squared-differences DIR and a dense local-correlation-coefficient DIR ${ }^{2}$. Afterwards, the previously created deformation vector field is applied to the structures on the pCT and new deformed structures are created on the sCT. Due to the

\footnotetext{
${ }^{2}$ Elekta $\mathrm{AB}$ and CMS Software, "ABAS: Intra-Patient Deformable Image Registration for Adaptive Radiotherapy - A White Paper," 2014.
}

limited field-of-view (FOV) of the CBCT, all deformed structures were clipped in cranio-caudal direction to the extent of the sCT.

The deformed structures (D) on the SCT were quantitatively compared to the manually delineated structures $(\mathrm{M})$ on the sCT with the following three metrics:

- The Dice similarity coefficient (DSC) defines the overlap of M and D volumes [31]:

$\mathrm{DSC}=\frac{2|\mathrm{M} \cap \mathrm{D}|}{|\mathrm{M}|+|\mathrm{D}|}$

- The mean surface distance $\left(\mathrm{d}_{\text {mean }}\right)$ represents the average of two directed mean surface distances $d_{a v g}$, where a point $X$ is compared to its nearest neighbor $\mathrm{Y}$ :

$\mathrm{d}_{\text {mean }}=\frac{\overrightarrow{\mathrm{d}_{\text {avg }}}(\mathrm{X}, \mathrm{Y})+\overrightarrow{\mathrm{d}_{\text {avg }}}(\mathrm{Y}, \mathrm{X})}{2}$

- The Hausdorff distance (HD) measures the maximum distance of two neighbored data points $\mathrm{x}$ and $\mathrm{y}$ in the two structures and is an indicator for the largest segmentation error:

$\mathrm{HD}(\mathrm{X}, \mathrm{Y})=\max _{\mathrm{x} \in \mathrm{X}} \min _{\mathrm{y} \in \mathrm{Y}}\|\mathrm{X}-\mathrm{y}\|$

A Wilcoxon signed-rank test was performed for all bilateral organs to test whether left- and right-sided organs showed statistically significant different structure properties. No p-value was larger than 0.05 and hence the analysis was performed for one combined structure. Finally, the same expert physician manually corrected the deformed structures on the sCT. The required time to perform these modifications was recorded.

\subsection{Analysis of the dosimetric accuracy}

To evaluate the dosimetric accuracy, dose distributions on pCT were re-calculated on sCT with identical beam-settings and compared to each other. Near-minimum, median and near-maximum doses $\mathrm{D}_{98}, \mathrm{D}_{50}$ and $\mathrm{D}_{2}$ of the gross tumor volume (GTV), $\mathrm{D}_{2}$ of the spinal cord, the mean dose $\left(D_{\text {mean }}\right)$ of the parotid glands as well as clinically relevant volumes $V_{j}$ for the brainstem, plexus, lung, heart, bladder and rectum that received more than a certain dose $\mathrm{j}$ (in Gray) were compared. For all relative quantities the value of the metric in the PCT represented the reference. No negative margins were applied for the dosimetric evaluation and outlier values were defined as results outside the interquartile-range (IQR) of $25-75 \%$ of all obtained results. Missing patient tissue on SCT, caused by a too small CBCT-FOV, was added by copying the external structure from pCT and subtracting the FOV of the CBCT, if necessary. The ED of the missing patient was overwritten with 1 [32]. Furthermore, $3 \mathrm{D}$ global gamma pass rates with criteria of $2 \% / 2 \mathrm{~mm}$ and $3 \% / 3 \mathrm{~mm}$ and a 3D point-wise dose difference analysis with a deviation threshold of $2 \%$ were determined between the dose distributions of the pCT and sCT. Low dose thresholds of 50\% (gamma analysis) and 90\% (dose difference 
analysis) of the prescription dose were used.

\section{Results}

\subsection{Analysis of image uncertainties}

In Fig. 1 representative axial slices of three arbitrary patients of the pCT (row (a)), the corresponding first fraction CBCT (row (b)) and the resulting SCT (row (c)) are displayed. Additionally, axial, coronal and sagittal slices of another three exemplary patients with common image artifacts are shown in the supplement Figs. S1-S3. For each of the three body regions, an improved assignment of image grey values to CT numbers is recognizable for the SCT compared to the CBCT which is confirmed by the absolute intensity volume histograms of the total CBCT scan volume presented in row (d) of Fig. 1. Deviations between SCT and pCT regarding the CT number maxima for lung tissue can be identified.

This observation is validated by the mean results for the ME and MAE
Table 3

Mean error (ME) and mean absolute error (MAE) in Hounsfield Units (HU) of the three body sites for the regions of interest (ROI) of the body contour $(-15 \mathrm{~mm}$ margin), lung ( $-10 \mathrm{~mm}$ margin), pelvic soft tissue $(-10 \mathrm{~mm}$ margin), pelvic bones $(-4 \mathrm{~mm}$ margin) and $\mathrm{H} \& \mathrm{~N}$ bones $(-2 \mathrm{~mm}$ margin). Values are displayed in terms of mean \pm standard deviation within a collective of 15 patients for each body region. The symbol * denotes significant differences $(\mathrm{p} \leq 0.05)$ identified in a two-tailed $t$ test between the mean ME and OHU.

\begin{tabular}{llll}
\hline Body site & ROI & ME (HU) & MAE (HU) \\
\hline H\&N & Body contour & $1.4 \pm 9.9$ & $77.2 \pm 12.6$ \\
& Bones & $24.0^{*} \pm 36.1$ & $163.0 \pm 42.6$ \\
Thorax & Body contour & $29.6^{*} \pm 30.0$ & $94.2 \pm 31.7$ \\
& Lung & $22.6^{*} \pm 29.3$ & $73.7 \pm 20.5$ \\
Pelvis & Body contour & $5.4^{*} \pm 4.6$ & $41.8 \pm 5.3$ \\
& Soft tissue & $0.5 \pm 8.4$ & $24.4 \pm 9.9$ \\
& Bones & $35.2^{*} \pm 19.5$ & $118.5 \pm 25.9$ \\
\hline
\end{tabular}
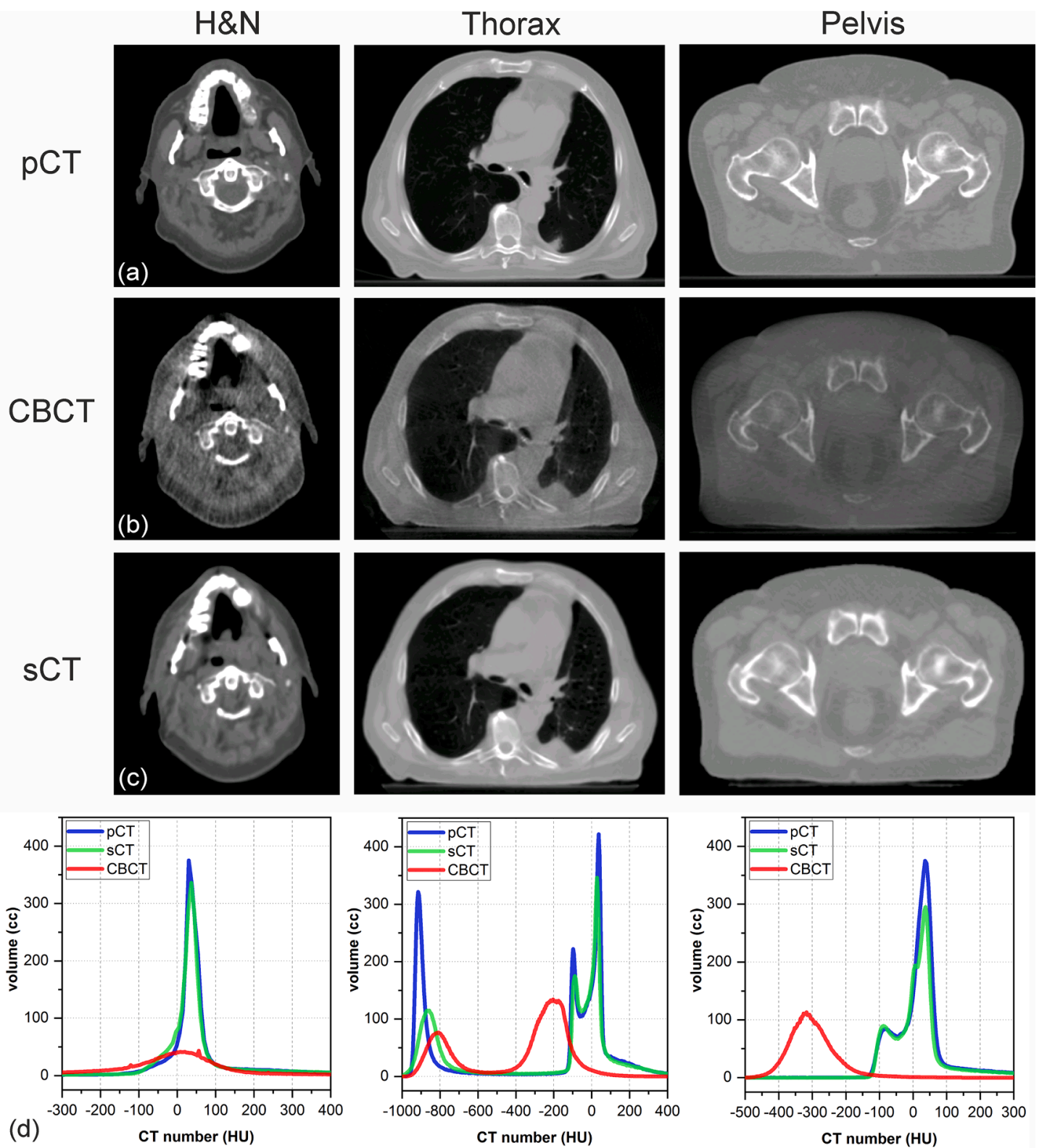

Fig. 1. Planning CT (pCT), cone-beam CT (CBCT) and synthetic CT (sCT) of three exemplary patients for the three body regions head and neck (H\&N), thorax and pelvis. Rows (a) to (c) represent the axial slices. Window level settings of [-350 550] Hounsfield units (HU), [-1000 600]HU and [-900 700]HU were applied to the images of the H\&N, thoracic and pelvic body region. Row (d) shows the absolute CT number distribution of the three image types for the body contour ROI minus a $15 \mathrm{~mm}$ margin. 
for the seven ROIs and the three body regions that are presented in Table 3. After sCT conversion, the ME were close to $0 \mathrm{HU}$ with $1.4 \mathrm{HU}$, $29.6 \mathrm{HU}, 5.4 \mathrm{HU}$ and the MAE resulted in $77.2 \mathrm{HU}, 94.2 \mathrm{HU}, 41.8 \mathrm{HU}$, respectively, for the body contour ROIs of pelvis, thorax and H\&N. The $\mathrm{SD}$ of the reference pCT image noise was 11.4HU. Inter-patient SD of ME and MAE were lower in all scenarios after SCT conversion. While the cycle-GAN performed best in the pelvic soft tissue ( $\mathrm{ME}=0.5 \pm 8.4 \mathrm{HU}$ ), the largest ME occurred for pelvic bony tissue with $35.2 \mathrm{HU}$, followed by the thoracic body contour ROI with 29.6HU. In general, all mean ME results were significantly different from $0 \mathrm{HU}$ except for the $\mathrm{H} \& \mathrm{~N}$ body contour ROI and the pelvic soft tissue ROI. The MAE was the largest for the H\&N bone ROI and pelvic bone ROI with $163.0 \mathrm{HU}$ and $118.5 \mathrm{HU}$, respectively.

\subsection{Segmentation accuracy analysis}

Table 4 displays the findings of the DSC, HD and $\mathrm{d}_{\text {mean }}$ between the deformed and manually generated structures for relevant OAR in the three body regions. Best average DSC results were $94.9 \%$ (lung), $90.5 \%$ (bladder), $85.9 \%$ (prostate) and $84.4 \%$ (heart). The highest inter-patient SD was obtained for plexus $(10.4 \%)$ that also had the lowest DSC (60.6\%). All average $d_{\text {mean }}$ values were below $3.5 \mathrm{~mm}$ except for the heart with $6.3 \mathrm{~mm}$. The mean HD was below $10 \mathrm{~mm}$ for the bilateral parotid gland, the spinal cord and the prostate. The maximum mean HD was obtained for the lung with $28.5 \pm 7.3 \mathrm{~mm}$. The required time for correcting one deformed structure set was $5.2 \pm 1.6 \mathrm{~min}$.

\subsection{Dosimetric accuracy analysis}

In Fig. 2 the relative differences in $\mathrm{D}_{98}, \mathrm{D}_{50}$ and $\mathrm{D}_{2}$ of the GTV for the three body regions are shown. All results on the sCT had mean dose differences of less than $1.7 \%$ when compared to the pCT. Except for the $\mathrm{D}_{98}$ in the H\&N body region, all median GTV doses are higher on sCT. One outlier was observed for the thoracic body region with an individual dose difference of $6.3 \%$ for $\mathrm{D}_{2}$ while the results for the body regions $\mathrm{H} \& \mathrm{~N}$ and pelvis showed maximum dose differences in DVH parameters of the target volume of $2.4 \%$ and a maximum SD of $0.7 \%$.

Eight dose-volume parameters for ten different OAR were compared for the three body regions. The related differences $\mathrm{V}_{\mathrm{i}}(\mathrm{sCT})-\mathrm{V}_{\mathrm{i}}(\mathrm{CT})$ are visualized in Fig. 3. The results of the sCT are in good agreement with the corresponding references on the pCT with mean deviation of less than $0.5 \%$ for all volume differences. Comparably large differences between doses on sCT and pCT were identified for the $\mathrm{D}_{2}$ of the spinal cord in the $\mathrm{H} \& \mathrm{~N}$ and thoracic regions and the $\mathrm{D}_{\text {mean }}$ of the parotid glands with mean values \pm IQR of $3.2 \pm 4.9 \%, 5.5 \pm 5.2 \%$ and $0.7 \pm 4.4 \%$, respectively.

The results of the 3D Gamma-dose analysis and the point-wise dose difference analysis are shown in Table 5. The 3D-Gamma pass rates for the $H \& N$, the thoracic and the pelvic body region were $98.6 \pm 1.0 \%$,

Table 4

Dice similarity coefficient (DSC), Hausdorff distance (HD) and mean surface distance $\left(\mathrm{d}_{\text {mean }}\right)$ of relevant organs at risk in terms of mean \pm standard deviation within the three body sites. The metrics are calculated between the manually delineated and deformed structures on the synthetic CT.

\begin{tabular}{lllll}
\hline Body site & Organ & DSC $(\%)$ & HD $(\mathrm{mm})$ & $\mathrm{d}_{\text {mean }}(\mathrm{mm})$ \\
\hline \multirow{2}{*}{ H\&N } & Brainstem & $73.8 \pm 7.3$ & $10.5 \pm 3.6$ & $3.5 \pm 1.2$ \\
& Parotid gland bilateral & $79.6 \pm 7.8$ & $9.9 \pm 7.1$ & $1.7 \pm 0.5$ \\
& Plexus bilateral & $60.6 \pm 10.4$ & $17.4 \pm 8.2$ & $2.6 \pm 0.9$ \\
& Spinal cord & $79.3 \pm 5.8$ & $5.0 \pm 1.6$ & $1.1 \pm 0.4$ \\
Thorax & Heart & $84.4 \pm 5.2$ & $22.5 \pm 5.5$ & $6.3 \pm 2.4$ \\
& Lung bilateral & $94.9 \pm 2.0$ & $28.5 \pm 7.3$ & $3.2 \pm 1.4$ \\
& Spinal cord & $81.8 \pm 3.1$ & $7.3 \pm 7.1$ & $1.1 \pm 0.3$ \\
& Bladder & $90.5 \pm 3.6$ & $15.3 \pm 9.2$ & $2.7 \pm 1.7$ \\
& Prostate & $85.9 \pm 3.3$ & $8.6 \pm 3.0$ & $2.2 \pm 0.7$ \\
& Rectum & $81.0 \pm 3.3$ & $14.3 \pm 7.2$ & $2.5 \pm 1.3$ \\
& Seminal vesicles & $66.7 \pm 8.3$ & $11.5 \pm 2.6$ & $1.9 \pm 0.4$ \\
\hline
\end{tabular}

$97.8 \pm 3.3 \%$ and $99.9 \pm 0.1 \%$ for a $3 \% / 3 \mathrm{~mm}$ gamma criterion and 95.0 $\pm 2.4 \%, 93.8 \pm 5.9 \%$ and $98.5 \pm 1.7 \%$ for a $2 \% / 2 \mathrm{~mm}$ gamma criterion. The highest deviations occurred at the patient outline of each body contour region and at the interface between lung and soft tissue. The pass rate of the point-wise dose difference analysis showed that on average $91.5 \%, 76.7 \%$ and $88.9 \%$ of all voxels had smaller deviations than $2 \%$ for the $H \& N$, thoracic and pelvic region, respectively.

\section{Discussion}

The ADMIRE AI software allows for sCT generation solely on the basis of daily CBCT and preserves the actual patient anatomy. Promising results were obtained throughout the segmentation and dosimetric analysis, proving the applicability in clinical routine with regard to crucial adaptive workflow steps.

Visually, image artifacts and low quality of the CBCT could be corrected by the sCT generation which resulted in a reliable assignment of CT numbers. The lowest ME/MAE values with the smallest SD for the body contour ROI and soft tissue ROI were obtained for the pelvic model with 5.4/41.8HU and $0.5 / 24.4 \mathrm{HU}$ which is linked to the larger number of patients for the training datasets of the cycle-GAN and the absence of air in the pelvic anatomy. Related cycle-GAN-based sCT studies reported similar values for the ME/MAE of the total body contour with -3 / 16.1HU [21] and -6/87HU [33]. The analysis of Kida et al. [30] which compared cycle-GAN generated sCT to deformed CT yielded mean HU values for the prostate and bladder of $19 \mathrm{HU}$ and $4 \mathrm{HU}$. However, a direct comparison remains difficult since all referenced studies differed from the presented work with regard to the size of the patient collective, the manufacturer-specific image acquisition parameters, the detailed architecture of the GAN and the training of the body site-specific models. Although the pelvic bone ROI exhibited the highest ME, the obtained value range for this high density region coincides with another image analysis study that investigated sCT created with a convolutional neural network (CNN) based on MRI [34]. No other analysis of a separate bone ROI of a CBCT-based sCT research study was found in the literature.

The sCT image quality analysis of the H\&N yielded ME/MAE values of the body contour and bone ROI of $1.4 / 77.2 \mathrm{HU}$ and $24 / 163 \mathrm{HU}$, respectively. These results were lower than those reported by Barateau et al. [35] with $17.1 / 82.4 \mathrm{HU}$ and $64.9 / 207.6 \mathrm{HU}$ that also used the Elekta XVI system for CBCT acquisition. Other studies using GAN for CBCT image processing presented values of $-6 \mathrm{HU}$ for the $\mathrm{ME}$ and 29.9HU and 51HU for the MAE of the body contour ROI [22,25]. The latter study by Maspero et al. also made use of the same CBCT image acquisition system but trained the single GAN with only 15 patients. Moreover, this was the only reference to state the ME/MAE of thorax SCT to be $-5 / 86 \mathrm{HU}$ [25] which is in a similar range to the presented results of the thoracic body contour ROI. The identified larger SD of up to $29 \mathrm{HU}$ for the thorax model might be caused by the inhomogeneity and hence different densities inside the lungs and breathing artifacts due to the longer scan times of the CBCTs compared to the pCT. Furthermore, it has to be noted that the different patient anatomy between the CT and SCT has a substantial influence on all presented dosimetric results.

Due to the general lack of literature about contour segmentation on synthetic images, all contouring metrics results of the presented study are indirectly compared to DIR- or DL-based segmentation approaches. Regarding the H\&N model, lowest consistency between manually delineated and DIR-propagated pCT structures on the SCT was obtained with mean DSCs between $60.6 \%$ and $79.3 \%$ and a comparably large SD which can be related to the smaller amount of patients in the training dataset of the H\&N model. Surveys investigating segmentation with DL or CNN techniques presented similar results [36-38] which support the general indication that anatomical properties of this particular tumor site are most difficult to process for DIR algorithms and already underlie larger inter-observer variability $[36,39]$ as well as daily positioning errors in case of brachial plexus [40].

The $\mathrm{d}_{\text {mean }}$ and HD of lung and heart fell short in comparison to all 


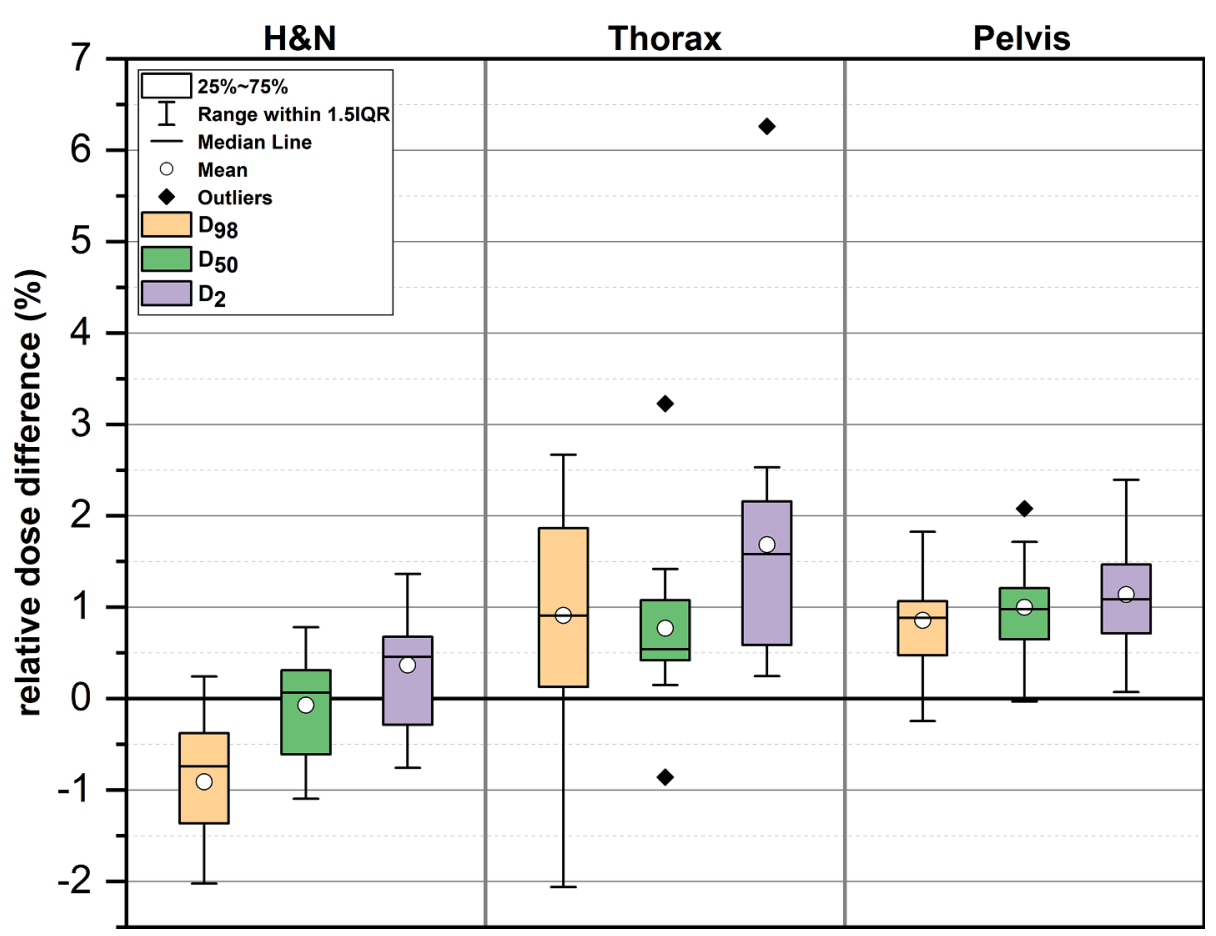

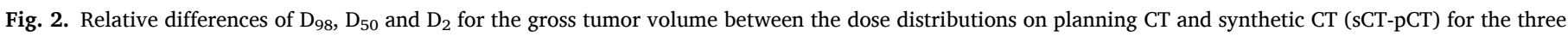

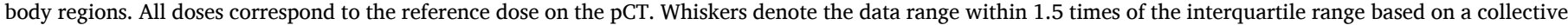
of 15 patients for each body region.
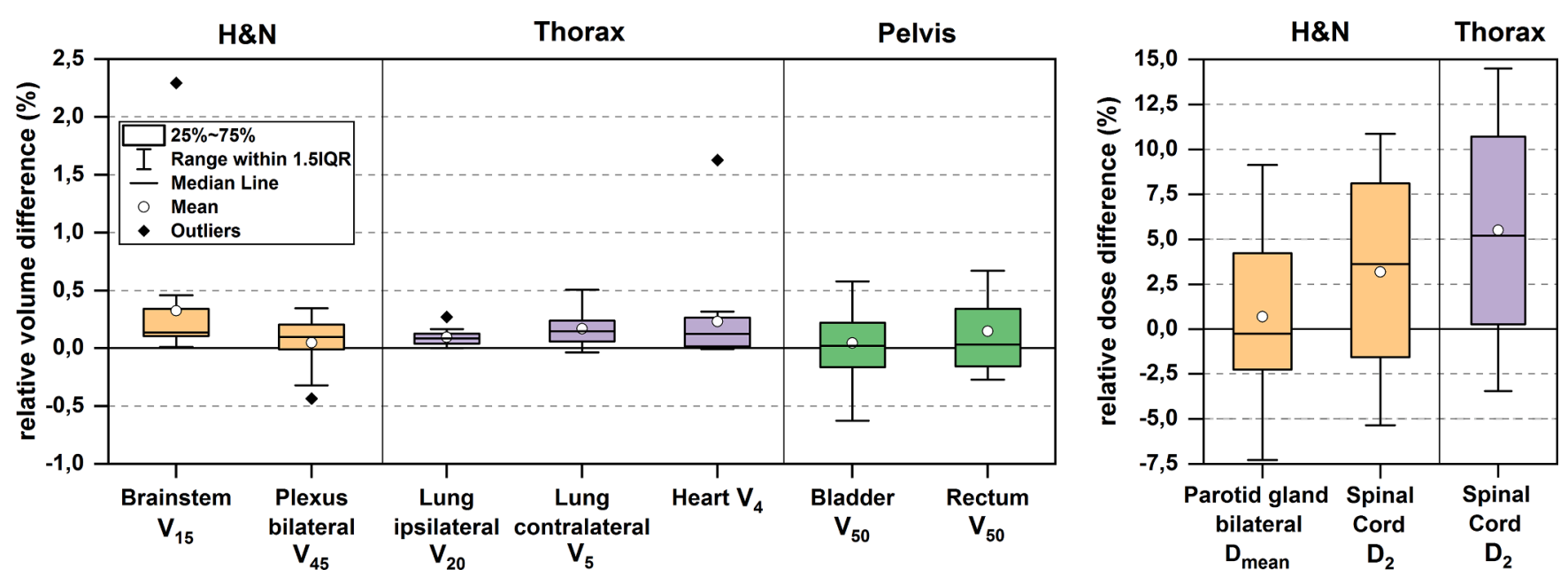

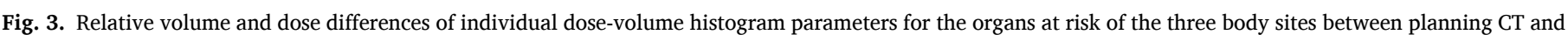

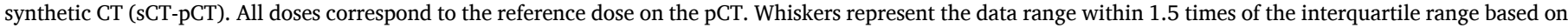
a collective of 15 patients for each body region. A reduced collective of 14 patients was evaluated for the plexus and parotid gland.

other structures among the three body regions. This was caused by the fact that the heart and the thoracic apex are typically located in the periphery of a lung CBCT scan where scatter artifacts could not be completely removed by the ADMIRE AI software. The insufficient contrast of the heart to the surrounding soft tissue additionally intensifies this effect. The DSC of $94.9 \%$ of the lung was comparable to DSC results of $95.5 \%-97.5 \%$ of a study evaluating DIR contours on 4D CT [41] but the DSC of the spinal cord stayed below the related results of 89-91\%.

The analysis of the pelvis segmentation model provided the most robust results, being in line with findings of other recent studies that evaluated DL or DIR approaches [42-44] and the well-known interobserver variability for prostate and seminal vesicles [45]. More than any other body region, the pelvic region is subject to daily varying organ filling and the potential presence of bowel gas degrades the results of DIR segmentation algorithms [46].

Overall, the ADMIRE intra-patient segmentation module was able to provide a complete image segmentation of a SCT within $30 \mathrm{~s}$. Manual modifications of a deformed structure set amounted to $5.2 \pm 1.6 \mathrm{~min}$ which yields a required time of maximum 8 min per complete segmentation of a daily sCT. This time range might be suitable for online ART but still needs to be further accelerated.

The dose calculation on the sCT showed good agreement with the reference dose on the PCT in case of the GTV with all IQR not exceeding $\pm 2.0 \%$ (Fig. 2). Although several studies using cycle-GAN algorithms for CBCT-based SCT generation have been conducted during the last 
Table 5

Pass rates of the 3D Gamma (3\%/3mm and $2 \% / 2 \mathrm{~mm}$ criteria) and dose difference analysis (point-wise difference $<2 \%$ ) calculated between the dose distributions on the synthetic CT and planning CT. Low dose thresholds of 50\% and $90 \%$ of the prescription dose were used, respectively. The results are reported as percentage mean \pm standard deviation of each 15 patients for the three body sites.

\begin{tabular}{llll}
\hline \multirow{2}{*}{ Body site } & \multicolumn{2}{l}{ Pass rates (\%) } \\
\cline { 2 - 4 } & $\begin{array}{l}\text { Gamma } \\
(3 \% / 3 \mathbf{m m})\end{array}$ & $\begin{array}{l}\text { Gamma } \\
(\mathbf{2 \% / 2 m m})\end{array}$ & Dose Difference (less than 2\%) \\
\hline H\&N & $98.6 \pm 1.0$ & $95.0 \pm 2.4$ & $91.5 \pm 4.3$ \\
Thorax & $97.8 \pm 3.3$ & $93.8 \pm 5.9$ & $76.7 \pm 17.3$ \\
Pelvis & $99.9 \pm 0.1$ & $98.5 \pm 1.7$ & $88.9 \pm 9.3$ \\
\hline
\end{tabular}

years, only a few complemented the image quality analysis with an additional dose evaluation. Regarding target volume dose metrics, two related studies reported dosimetric differences between the sCT and CT with a comparable accuracy to our study. A $\mathrm{V}_{95}$ of $0.2-0.7 \%$ for the $\mathrm{H} \& \mathrm{~N}$ body site [35] and an absolute difference of $D_{98}, D_{50}$ and $D_{2}$ of -1 to 0 Gy for the pelvic body site [30] were obtained, respectively. For the thoracic body region, $\mathrm{D}_{98}$ showed the largest deviations and for the $\mathrm{D}_{50}$ and $\mathrm{D}_{2}$ outliers were observed which can be attributed to remaining uncertainties due to the breathing pattern. A study about artifactcorrected and deformable registered CBCT presented a mean deviation from the pCT for $\mathrm{D}_{98}$ (PTV) of less than 0.5 Gy [47], which equals a relative deviation of less than $0.76 \%$ compared to our result of $0.91 \%$. Furthermore, small inaccuracies in the low CT number region of the CTED-calibration curve have a bigger impact on the relative ED. This condition is related to the steep gradient of the calibration curve in the range between $-950 \mathrm{HU}$ and $85 \mathrm{HU}$ in contrast to the robust CT number assignment within the soft tissue region.

For all volume differences of the OAR between the sCT and pCT presented in Fig. 3 the mean deviations were less than $0.5 \%$, but showed a tendency of a dose overestimation on the sCT. A reason for this trend might be caused by the slightly higher HU values expressed by the positive ME. The deviations for the OARs were particularly small for the thorax model and demonstrated a good reliability of the dose calculations with no IQR being larger than $0.25 \%$ of the relative dose difference. Only for the rectum and bladder, the volume differences fluctuated more between $\pm 1 \%$ which could also be caused by varying organ fillings. Similar deviation ranges for pelvic OAR were observed by related research on CBCT-based [33] and MRI-based [24] sCT generation methods. The spinal cord of both H\&N and thoracic body site and the parotid gland showed the largest deviations amongst all OAR dose parameters for both the $\mathrm{H} \& \mathrm{~N}$ and thoracic region, having dose difference values of up to $14.5 \%$. Comparably large dose deviations were also obtained by a DIR-based CBCT correction approach with $-5.55 \% \pm$ $14.3 \%$ for the thoracic spinal cord [48]. For the H\&N body site, absolute mean dose differences for the parotid gland and the spinal cord were reported as $0.05-0.07 \mathrm{~Gy}$ and $0.42 \mathrm{~Gy}$ [35] which compares to our results by a factor of approximately 0.5 . This discrepancy arises from the fact that the related study used rigid and deformable image registrations between the CBCT and PCT to minimize the negative effects of anatomic variations. In general, heterogeneities due to surrounding bone material in low dose regions potentially impair dosimetric accuracy and thus the spinal cord is more sensitive to dosimetric changes than other OAR. Furthermore, larger dose deviations in the region of the parotid gland can occur due to the systematic anatomical differences between the pCT and sCT within the presented study.

The analysis of the 3D gamma pass rates for the pelvic body site with a $2 \% / 2 \mathrm{~mm}$ and $3 \% / 3 \mathrm{~mm}$ criterion yielded consistent results with no single value being less than $95 \%$ and a SD of $0.1 \%$ for the $3 \% / 3 \mathrm{~mm}$ criterion. The results agreed with the findings of Kurz et al. of $96 \%$ and $100 \%$ [33] but stayed below the mean pass rate of $99.5 \%$ for the $2 \%$ / $2 \mathrm{~mm}$ criterion of a U-Net SCT generation method presented by Landry et al. [45]. The voxel-wise $2 \%$ dose difference pass rate (DD-PR) was $88.91 \%$ and, supported by visual comparisons of dose distributions within the single patients' anatomies, revealed that a large proportion of voxels had a dose difference around $2 \%$ in the central soft tissue region. Kurz et al. presented a $2 \%$ DD-PR of $100 \%$ [33] but used a different GAN architecture and a collapsed-cone algorithm for VMAT plan calculations.

The mean gamma pass rate for a $2 \% / 2 \mathrm{~mm}$ criterion for the $\mathrm{H} \& \mathrm{~N}$ patients was over $95 \%$. Three related studies working with GAN-based SCT reported values of 98.1\% [35], 98.4\% [22] and 95.3\% [49] but were only indirectly relatable on account of differing dose thresholds or various types of reference images. In this sCT study, most dosimetric deviations were found in the regions of air cavities or PTV parts close to the daily varying patient outline. This lead to a small proportion of voxels having larger dosimetric deviations and was also implied by the largest $2 \%$ DD-PR of $91.5 \%$ for the H\&N body site. Only one study reported the mean dose difference of GAN-processed CBCT with values of $0.1 \pm 0.5 \%[25]$.

For the thoracic region, the gamma analysis yielded the lowest results with a SD of up to $5.9 \%$ which can be related to several outliers and the aforementioned characteristics of the thorax CT-ED-calibration. Additionally, gamma pass rates for a threshold of $10 \%$ of the prescription dose were calculated for the thoracic region to be $93.4 \pm 4.9 \%(2 \%)$ $2 \mathrm{~mm})$ and $96.0 \pm 3.8 \%(3 \% / 3 \mathrm{~mm})$ and differed from the obtained results by Maspero et al. by $-1.5 \%$ and $-2.2 \%$ [25]. The same study reported mean dose differences with the same dose threshold of $90 \%$ to be $0.2 \pm 0.9 \%$ which is, based on indirect comparison, better than the obtained low 2\% DD-PR of $76.7 \%$ for the thorax body site of this sCT study. Given the implementation of masking truncated CBCT in the GAN architecture and the additional procedure of a rescan CT presented by the related sCT study [25], the gamma and dose difference analysis of this sCT study still revealed promising results and helped to identify the main regions (high dose gradients, soft tissue-lung interface and cropped FOV) in the thorax that substantially complicate image processing and dose calculation. To our knowledge, no other study except the one by Maspero targeted the dose calculation on thorax CBCT processed by DL approaches so far. Thus, our results can serve as a basis to further improve daily imaging protocols and training of sCT models.

Since the entire adaptive treatment process is integrated in the research interface of one planning system and the dosimetric results were within clinical tolerances, a crucial step towards the clinical implementation of ART on standard conventional linac was made. The presented methods have the potential for automating essential but timeconsuming treatment components and form the basis for adapting the initial treatment plan to the actual daily patient anatomy. However, quality assurance for every workflow step is mandatory prior to the use in clinical routine especially with regard to detecting possible outliers. For future clinical applications, a larger FOV would be beneficial for the thoracic and $\mathrm{H} \& \mathrm{~N}$ region to detect outline changes or additional positioning uncertainties. Besides of an increased daily imaging dose, a new training of the cycle-GAN model could further improve these current shortcomings, also with regard to the implementation of specific image characteristics like high density implants or tracheostomas.

\section{Conclusion}

The presented results demonstrate that daily СВСТ datasets were successfully processed by the ADMIRE cycle-GAN-based correction approach to generate sCT data with image properties close to a pCT for the body regions of $\mathrm{H} \& \mathrm{~N}$, thorax and pelvis. The CT numbers of the SCT were accurate enough to provide reliable dose calculations but anatomical regions with low soft tissue contrast still require special caution. Hence, the proposed method proved principal feasibility of all crucial workflow steps of a potential CBCT-based ART on a conventional linac within one software environment. 


\section{Acknowledgement}

The authors would like to thank Gustav Meedt, Nicolette O'Connell, Jiaofeng $\mathrm{Xu}$ and Daniel Thill from Elekta $\mathrm{AB}$ for their continuous support of this project. The research software ADMIRE was provided by Elekta and implemented in a Monaco research version. The project was partially funded by an Elekta AB research grant.

\section{Appendix A. Supplementary data}

Supplementary data to this article can be found online at https://doi. org/10.1016/j.ejmp.2020.11.007.

\section{References}

[1] Moller DS, et al. Adaptive radiotherapy for advanced lung cancer ensures target coverage and decreases lung dose. Radiother Oncol 2016;121(1):32-8. https://doi. org/10.1016/j.radonc.2016.08.019.

[2] Qin A, Sun Y, Liang J, Yan D. Evaluation of online/offline image guidance/ adaptation approaches for prostate cancer radiation therapy. Int J Radiat Oncol Biol Phys 2015;91(5):1026-33. https://doi.org/10.1016/j.ijrobp.2014.12.043.

[3] Jensen AD, Nill S, Huber PE, Bendl R, Debus J, Munter MW. A clinical concept for interfractional adaptive radiation therapy in the treatment of head and neck cancer. Int J Radiat Oncol Biol Phys 2012;82(2):590-6. https://doi.org/10.1016/j. ijrobp.2010.10.072.

[4] Qin Y, Zhang F, Yoo DS, Kelsey CR, Yin FF, Cai J. Adaptive stereotactic body radiation therapy planning for lung cancer. Int J Radiat Oncol Biol Phys 2013;87 (1):209-15. https://doi.org/10.1016/j.ijrobp.2013.05.008.

[5] Stankiewicz M, Li W, Rosewall T, Tadic T, Dickie C, Velec M. Patterns of practice of adaptive re-planning for anatomic variances during cone-beam CT guided radiotherapy. Technical Innovations \& Patient Support in Radiation Oncology 2019;12:50-5. https://doi.org/10.1016/j.tipsro.2019.10.003.

[6] Sonke JJ, Aznar M, Rasch C. Adaptive radiotherapy for anatomical changes. Semin Radiat Oncol 2019;29(3):245-57. https://doi.org/10.1016/j. semradonc.2019.02.007.

[7] Poludniowski GG, Evans PM, Webb S. Cone beam computed tomography number errors and consequences for radiotherapy planning: an investigation of correction methods. Int J Radiat Oncol Biol Phys 2012;84(1):e109-14. https://doi.org/ 10.1016/j.ijrobp.2012.02.019.

[8] Disher B, Hajdok G, Wang A, Craig J, Gaede S, Battista JJ. Correction for 'artificial electron disequilibrium due to cone-beam CT density errors: implications for online adaptive stereotactic body radiation therapy of lung. Phys Med Biol 2013;58 (12):4157-74. https://doi.org/10.1088/0031-9155/58/12/4157.

[9] Schulze R, et al. Artefacts in СВCT: a review. Dentomaxillofac Radiol 2011;40(5): 265-73. https://doi.org/10.1259/dmfr/30642039.

[10] Giacometti V, Hounsell AR, McGarry CK. A review of dose calculation approaches with cone beam CT in photon and proton therapy. Phys Med 2020;76:243-76. https://doi.org/10.1016/j.ejmp.2020.06.017.

[11] de Smet M, Schuring D, Nijsten S, Verhaegen F. Accuracy of dose calculations on $\mathrm{kV}$ cone beam CT images of lung cancer patients. Med Phys 2016;43(11):5934. https://doi.org/10.1118/1.4964455.

[12] Fotina I, Hopfgartner J, Stock M, Steininger T, Lutgendorf-Caucig C, Georg D. Feasibility of CBCT-based dose calculation: comparative analysis of HU adjustment techniques. Radiother Oncol 2012;104(2):249-56. https://doi.org/10.1016/j. radonc.2012.06.007.

[13] Schröder L, Stankovic U, Remeijer P, Sonke J-J. Evaluating the impact of conebeam computed tomography scatter mitigation strategies on radiotherapy dose calculation accuracy. Physics and Imaging in Radiation Oncology 2019;10:35-40. https://doi.org/10.1016/j.phro.2019.04.001.

[14] Hansen DC, et al. ScatterNet: A convolutional neural network for cone-beam CT intensity correction. Med Phys 2018;45(11):4916-26. https://doi.org/10.1002/ mp.13175.

[15] Jiang Y, et al. Scatter correction of cone-beam CT using a deep residual convolution neural network (DRCNN). Phys Med Biol 2019;64(14). https://doi.org/10.1088/ 1361-6560/ab23a6.

[16] Cole AJ, Veiga C, Johnson U, D'Souza D, Lalli NK, McClelland JR. Toward adaptive radiotherapy for lung patients: feasibility study on deforming planning CT to CBCT to assess the impact of anatomical changes on dosimetry. Phys Med Biol 2018;63 (15). https://doi.org/10.1088/1361-6560/aad1bb.

[17] Branchini M, et al. Validation of a method for "dose of the day" calculation in headneck tomotherapy by using planning ct-to-MVCT deformable image registration. Phys Med 2017;39:73-9. https://doi.org/10.1016/j.ejmp.2017.05.070.

[18] Brock KK. Adaptive radiotherapy: moving into the future. Semin Radiat Oncol 2019;29(3):181-4. https://doi.org/10.1016/j.semradonc.2019.02.011.
[19] Thompson RF, et al. Artificial intelligence in radiation oncology: A specialty-wide disruptive transformation? Radiother Oncol 2018;129(3):421-6. https://doi.org/ 10.1016/j.radonc.2018.05.030.

[20] Li Y, et al. A preliminary study of using a deep convolution neural network to generate synthesized CT images based on CBCT for adaptive radiotherapy of nasopharyngeal carcinoma. Phys Med Biol 2019;64(14). https://doi.org/10.1088/ 1361-6560/ab2770.

[21] Harms J, et al. Paired cycle-GAN-based image correction for quantitative conebeam computed tomography. Med Phys 2019. https://doi.org/10.1002/mp.13656.

[22] Liang X, et al. Generating synthesized computed tomography (CT) from cone-beam computed tomography (CBCT) using CycleGAN for adaptive radiation therapy. Phys Med Biol 2019;64(12). https://doi.org/10.1088/1361-6560/ab22f9.

[23] Mori S, Hirai R, Sakata Y. Using a deep neural network for four-dimensional CT artifact reduction in image-guided radiotherapy. Phys Med 2019;65:67-75. https://doi.org/10.1016/j.ejmp.2019.08.008.

[24] Maspero M, et al. Dose evaluation of fast synthetic-CT generation using a generative adversarial network for general pelvis MR-only radiotherapy. Phys Med Biol 2018;63(18). https://doi.org/10.1088/1361-6560/aada6d.

[25] Maspero M, et al. A single neural network for cone-beam computed tomographybased radiotherapy of head-and-neck, lung and breast cancer. Physics and Imaging in Radiation Oncology 2020;14:24-31. https://doi.org/10.1016/j. phro.2020.04.002.

[26] Zhu J, Park T, Isola P, Efros AA. "Unpaired Image-to-Image Translation Using Cycle-Consistent Adversarial Networks," in: 2017 IEEE International Conference on Computer Vision (ICCV), 22-29 Oct. 2017 2017, pp. 2242-2251, doi: 10.1109/ ICCV.2017.244.

[27] Xu J, Han X, Hibbard L, Willcut V. A Deep Convolutional Neural Network for ConeBeam CT Artefacts Reduction: SU-K-FS4-14. Med Phys 2017;44(6):3011-2.

[28] J. P. Cohen, M. Luck, and S. Honari, "Distribution Matching Losses Can Hallucinate Features in Medical Image Translation," in: Medical Image Computing and Computer Assisted Intervention - MICCAI 2018, (Lecture Notes in Computer Science, 2018, ch. Chapter 60, pp. 529-536.

[29] Wolterink JM, Dinkla AM., Savenije M.HF, Seevinck PR, van den Berg CAT, Išgum I. "Deep MR to CT Synthesis Using Unpaired Data," Cham, 2017: Springer International Publishing, in Simulation and Synthesis in Medical Imaging, pp. 1423, doi: 10.1007/978-3-319-68127-6_2

[30] Kida S, et al. Visual enhancement of Cone-beam CT by use of CycleGAN. Med Phys 2020;47(3):998-1010. https://doi.org/10.1002/mp.13963.

[31] Dong X, et al. Automatic multiorgan segmentation in thorax CT images using Unet-GAN. Med Phys 2019;46(5):2157-68. https://doi.org/10.1002/mp.13458.

[32] van Zijtveld M, Dirkx M, Heijmen B. Correction of conebeam CT values using a planning CT for derivation of the "dose of the day". Radiother Oncol 2007;85(2): 195-200. https://doi.org/10.1016/j.radonc.2007.08.010.

[33] Kurz C, et al. CBCT correction using a cycle-consistent generative adversarial network and unpaired training to enable photon and proton dose calculation. Phys Med Biol 2019. https://doi.org/10.1088/1361-6560/ab4d8c.

[34] Fu J, et al. Deep learning approaches using 2D and 3D convolutional neural networks for generating male pelvic synthetic computed tomography from magnetic resonance imaging. Med Phys 2019;46(9):3788-98. https://doi.org/ 10.1002/mp.13672.

[35] Barateau A, et al. Comparison of CBCT-based dose calculation methods in head and neck cancer radiotherapy: from Hounsfield unit to density calibration curve to deep learning. Med Phys 2020. https://doi.org/10.1002/mp.14387.

[36] van Dijk LV, et al. Improving automatic delineation for head and neck organs at risk by Deep Learning Contouring. Radiother Oncol 2020;142:115-23. https://doi. org/10.1016/j.radonc.2019.09.022.

[37] Ibragimov B, Xing L. Segmentation of organs-at-risks in head and neck CT images using convolutional neural networks. Med Phys 2017;44(2):547-57. https://doi. org/10.1002/mp.12045.

[38] Liang S, et al. Deep-learning-based detection and segmentation of organs at risk in nasopharyngeal carcinoma computed tomographic images for radiotherapy planning. Eur Radiol 2019;29(4):1961-7. https://doi.org/10.1007/s00330-0185748-9.

[39] Brouwer CL, et al. 3D Variation in delineation of head and neck organs at risk. Radiat Oncol 2012;7:32. https://doi.org/10.1186/1748-717X-7-32.

[40] Yang J, et al. Automatic contouring of brachial plexus using a multi-atlas approach for lung cancer radiation therapy. Practical Radiation Oncology 2013;3(4): e139-47. https://doi.org/10.1016/j.prro.2013.01.002.

[41] Hardcastle N, van Elmpt W, De Ruysscher D, Bzdusek K, Tome WA. Accuracy of deformable image registration for contour propagation in adaptive lung radiotherapy. Radiat Oncol 2013;8:243. https://doi.org/10.1186/1748-717X-8243.

[42] Wong J, et al. Comparing deep learning-based auto-segmentation of organs at risk and clinical target volumes to expert inter-observer variability in radiotherapy planning. Radiother Oncol 2019;144:152-8. https://doi.org/10.1016/j. radonc. 2019.10.019.

[43] Sharp G, et al. "Vision 20/20: perspectives on automated image segmentation for radiotherapy," (in eng). Med Phys 2014;41(5). https://doi.org/10.1118/ 1.4871620 . 
[44] Woerner AJ, Choi M, Harkenrider MM, Roeske JC, Surucu M. Evaluation of Deformable Image Registration-Based Contour Propagation From Planning CT to Cone-Beam CT. p. 1533034617697242 Technol Cancer Res Treat 2017. https:// doi.org/10.1177/1533034617697242.

[45] [45] T. Nyholm et al., "Variability in prostate and seminal vesicle delineations defined on magnetic resonance images, a multi-observer, -center and -sequence study," Radiat Oncol, vol. 8, p. 126, May 24 2013, doi: 10.1186/1748-717X-8-126.

[46] Berenguer R, et al. The influence of the image registration method on the adaptive radiotherapy. A proof of the principle in a selected case of prostate IMRT. Phys Med 2018;45:93-8. https://doi.org/10.1016/j.ejmp.2017.12.007.
[47] Thing RS, Bernchou U, Hansen O, Brink C. Accuracy of dose calculation based on artefact corrected Cone Beam CT images of lung cancer patients. Physics and Imaging in Radiation Oncology 2017;1:6-11. https://doi.org/10.1016/j. phro.2016.11.001.

[48] Yuan Z, Rong Y, Benedict SH, Daly ME, Qiu J, Yamamoto T. "Dose of the day" based on cone beam computed tomography and deformable image registration for lung cancer radiotherapy. Journal of Applied Clinical Medical Physics 2019;21(1): 88-94. https://doi.org/10.1002/acm2.12793.

[49] M. Maspero et al., "CBCT-to-CT synthesis with a single neural network for headand-neck, lung and breast cancer adaptive radiotherapy," arXiv preprint arXiv: 1912.11136, 2019 